\title{
On the shape of barchan dunes
}

\author{
Klaus Kroy, Sebastian Fischer and Benedikt Obermayer \\ Hahn-Meitner Institut, Glienicker Straße 100, 14109 Berlin, Germany
}

\begin{abstract}
Barchans are crescent-shaped sand dunes forming in aride regions with unidirectional wind and limited sand supply. We report analytical and numerical results for dune shapes under different environmental conditions as obtained from the so-called 'minimal model' of aeolian sand dunes. The profiles of longitudinal vertical slices (i.e. along the wind direction) are analyzed as a function of wind speed and sand supply. Shape transitions can be induced by changes of mass, wind speed and sand supply. Within a minimal extension of the model to the transverse direction the scale-invariant profile of transverse vertical cuts can be derived analytically.
\end{abstract}

E-mail: kroy@hmi.de

\section{Introduction}

The interaction of turbulent wind with sand is known to create a surprisingly rich variety of sand structures in many places on Earth and also on other planets. Among the most symmetric structures are isolated heaps and barchan dunes. For a schematic sketch that introduces some of the terminology see figure 1. By identifying and isolating the mechanisms that are essential for dune formation, a mathematical minimal model could recently be formulated $[15,16]$, and was shown to reproduce generically field observations reporting systematic shape variations [20]. In particular, shape transitions from dunes (with slipface) to smooth heaps as a function of sand mass were predicted $[1,15,16]$. The model adapts, combines, and extends earlier theoretical developments in turbulence $[12,26]$ and aeolian sand transport $[19,22]$ into a consistent mathematical description, which allows to identify a weak spontaneous symmetry breaking of the turbulent air flow as the origin of the growth instability giving rise to structure formation. It moreover pinpoints the mutual competition of this symmetry breaking with saturation transients in the sand flux as the basic mechanism responsible for shape selection as a function of size and environmental parameters such as density of the air, wind speed or sand supply. The characteristic length scale provided by the saturation transients is called the saturation length $\ell_{\mathrm{s}}$ $[15,16,22]$. Due to the ballistic grain motion, it can be much larger than the grain diameter $d\left(\approx 10^{-4} \mathrm{~m}\right)$, which is the only elementary scale in the problem, and can thus compete with the turbulent symmetry breaking to cause scale dependence and shape transitions of dunes that are of the order of $10^{2} \mathrm{~m}$ long.

Below, we address some consequences of (broken) scale invariance by analyzing the predicitions of the minimal model for the longitudinal shape of a barchan as a function of environmental parameters. The next section reviews the major parameter dependencies of $\ell_{\mathrm{S}}$ and deduces some immediate consequences for shape similarities. Section 3 reports a systematic numerical study of the influence of dune size, wind 


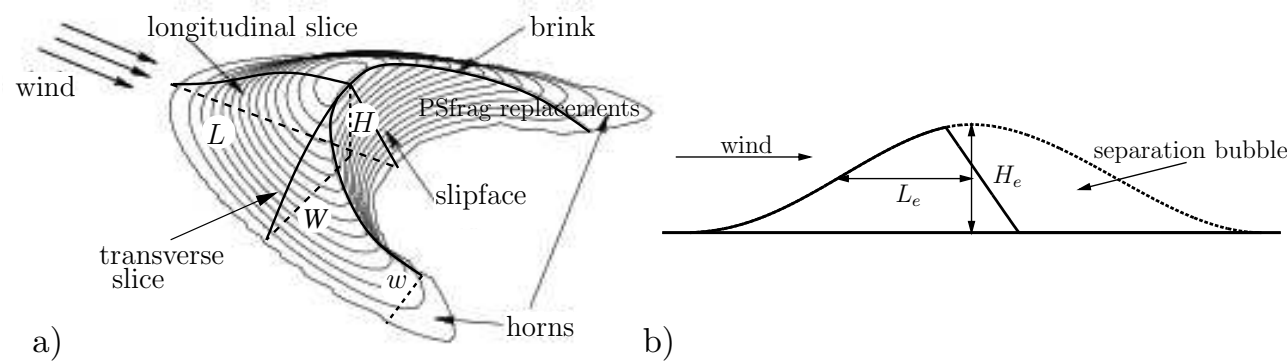

Figure 1. a) Sketch of a barchan dune introducing some terminology. b) Longitudinal cut along the symmetry plane. The region of flow separation is modelled by a smooth continuation of the dune profile [16] and the shear stress on the back of the dune is calculated as for a heap with the shape of the envelope (dune and separation bubble) with height $H_{\mathrm{e}}$ and characteristic length $L_{\mathrm{e}}$.

speed and sand supply on the longitudinal shape. Finally, in section 4, we extend the minimal model to the transverse direction. We close with a brief conclusion.

\section{Similarity hypothesis}

With only one characteristic length $\ell_{\mathrm{s}}$ in the problem, one expects that shapes of dunes of various size and under various environmental conditions can be related to each other by similarity transformations. This immediate consequence of the minimal model $[15,16]$ is supported by careful field measurements [20] and, more recently, by laboratory experiments with submarine dunes $[7,8,10,14]$. If this promising evidence could be further validated in future work, submarine structures forming in unidrectional flows, which were formerly identified as ripples $[3,4]$ on the basis of their superficial similarity to aeolian ripples, would actually have to be regarded as proper dunes. This could tremendously facilitate the experimental study of dunes and ultimately allow us to draw conlcusions about kilometer-sized dunes on Earth and possibly on other planets from analyzing centimeter-sized submarine dunes in the lab. However, some caution is needed in postulating simple one-to-one relations of similar structures under completely different environmental conditions. Arguably, the minimal model, which has the form of a concise hydrodynamic description of sand transport in turbulent shear flow [15], could well be applicable outside the realm of validity of the expressions and values derived for its kinetic coefficients, such as $\ell_{\mathrm{s}}$, so that similar dune structures may not always be reliable indicators for similar elementary transport processes.

For any fixed given ratio $\tau / \tau_{\mathrm{t}}$ of the wind shear stress $\tau$ to the threshold shear stress $\tau_{\mathrm{t}}$ for the mobilization of grains by the wind, the saturation length is predicted $[15,22]$ to scale linearly in $\tau_{\mathrm{t}}$ divided by the gravitational acceleration $g$ and the atmospheric density $\rho_{\mathrm{a}}$. According to conventional estimates from simple arguments for the aerodynamics on the grain scale, $\tau_{\mathrm{t}}=\theta \rho^{\prime} g d$ [2], i.e. $\ell_{\mathrm{s}}$ scales linearly in the grain diameter $d$ as required by dimensional analysis and also linearly in $\rho^{\prime} / \rho_{\mathrm{a}}$, the immersed over the atmospheric density. The dimensionless (generalised) Shields parameter $\theta[2,24]$ accounts for the complicated interactions of the hopping grains with the air and the bed. It usually can be taken to be a phenomenological constant for aeolian sand transport on Earth. The density ratio $\rho^{\prime} / \rho_{\text {a }}$ ranges from about $2 \cdot 10^{5}$ for typical conditions on Mars, through $2.2 \cdot 10^{3}$ on Earth and about 50 on Venus, 


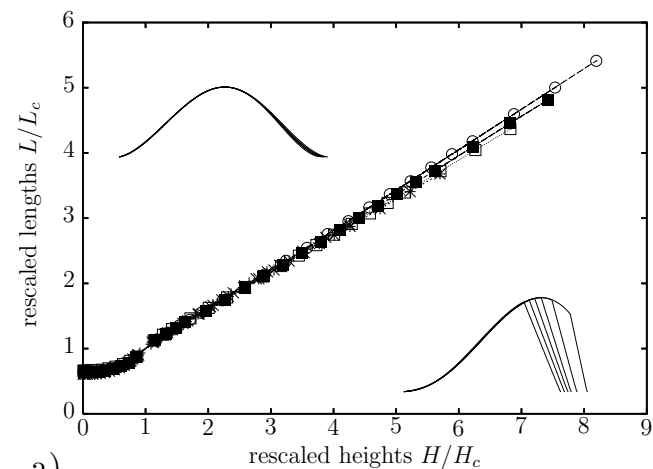

a)

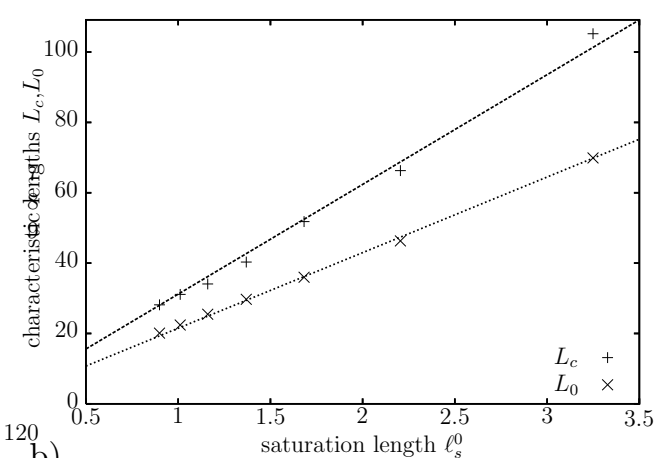

b)

Figure 2. a) Height-length relations of windward longitudinal steady-state profiles for various "masses" $\left(1-676 \mathrm{~m}^{2}\right)$ and wind speeds $\left(\tau^{0} / \tau_{\mathrm{t}}=1.4,1.6\right.$, $1.8,2.0,2.2,2.4,2.6)$ collapse onto a universal curve if heights and lengths are normalized to their values $H_{\mathrm{c}}, L_{\mathrm{c}}$ at the shape transition. Insets: The windward profiles of all dunes and heaps are of the functional form (2). b) The transition length $L_{\mathrm{c}}$ and the minimum heap length $L_{0} \approx 0.7 L_{\mathrm{c}}$ scale linearly in $\ell_{\mathrm{s}}^{0}$.

to 1.7 for submarine flows and can thus dramatically amplify the effect of broken scale invariance beyond the grain scale $d$. Typical grain sizes found in dunes are estimated to be roughly the same on Mars and Earth [6], and similar (or slightly smaller) grain sizes have been employed in laboratory experiments with submarine dunes $[3,4,10]$. One can therefore roughly estimate the ratios of the various $\tau_{\mathrm{t}}$ as Mars/Earth/Venus/water $\approx 10 / 1 / 1 / 0.5$, respectively, if potential differences in the Shields parameter are disregarded [13]. Accordingly, we expect $\ell_{\mathrm{S}}$ ratios (and thus size ratios, if size is measured relative to the corresponding grain size) of

$$
\text { Mars/Earth/Venus/Water } \approx 10^{5} / 10^{3} / 10^{1} / 1
$$

for dunes of the same shape, with the same $\tau / \tau_{\mathrm{t}}$, and with the same degree of saturation of the upwind sand flux. This implies that asymptotically large dunes, which should have a universal scale-invariant shape, are most easily produced in water $[3,4,10]$. The similarity rule (1) is well supported by a comparison of aeolian and submarine dunes $[10,14,20]$. However, there may be some evidence $[5,25]$ that it overestimates the scale factor between dunes on Earth and on Mars. This could be a consequence of the considerable differences in atmospheric densities and corresponding differences in the dominant sand transport mechanisms and Shields parameters on both planets [18].

\section{Longitudinal profiles}

To make the content of the similarity hypothesis more palpable, we report here results for the longitudinal profiles under various wind and influx conditions obtained by numerical solution of the minimal model. To simplify the notation we introduce the reference shear stress $\tau^{0}$ over the flat bed and corresponding notation for the saturation length $\ell_{\mathrm{s}}^{0} \equiv \ell_{\mathrm{s}}\left(\tau^{0}\right)$, and the saturated sand flux $q_{\mathrm{s}}^{0} \equiv q_{\mathrm{s}}\left(\tau^{0}\right)$. Figure 2a) combines steady-state solutions (influx $=$ outflux) for various sizes and wind speeds. The insets demonstrate exemplarily that all windward profiles are well represented by

$$
h(x) \approx H_{\mathrm{e}} \cos ^{\alpha}\left(x / L_{\alpha}\right), \quad L_{\alpha} \equiv L_{\mathrm{e}} / \arccos \left(2^{-1 / \alpha}\right)
$$




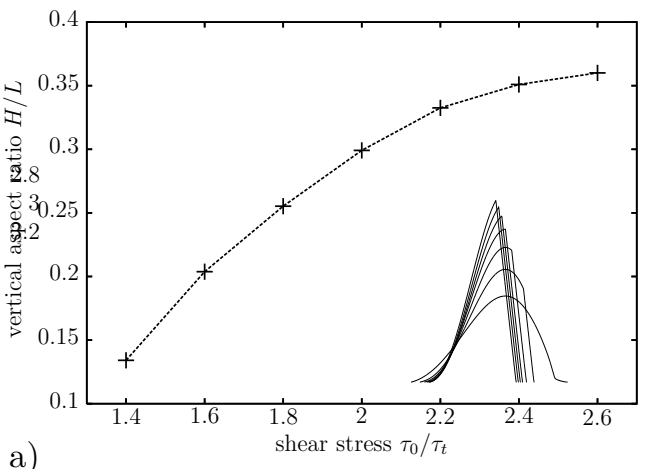

a)

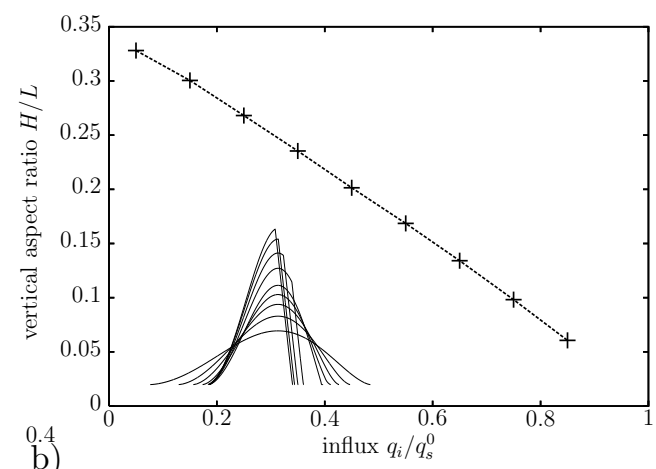

b)

Figure 3. Longitudinal vertical profiles of given mass $\left(225 \mathrm{~m}^{2}\right)$ : a) for varying wind shear stress (steady-state profiles); b) for given reference shear stress $\tau^{0} / \tau_{\mathrm{t}}=2.2$ but varying influx. Again, all windward profiles obey equation (2).

with $\alpha \approx 3.0$ for dunes and $\alpha \approx 1.8$ for heaps $\left(H_{\mathrm{e}}, L_{\mathrm{e}}\right.$ are defined in figure $\left.1 \mathrm{~b}\right)$. Such collapse of the windward profiles is strongly supported by field data [1,20]. Moreover, the height-length relations for diverse masses and wind speeds can be superimposed on a single master curve by rescaling lengths and heights by their values $H_{\mathrm{c}}, L_{\mathrm{c}}$ at the shape transition. The data points at the lower left indicate that in units of $\ell_{\mathrm{s}}^{0}$ all heaps have roughly the same length $L_{0} \leq L \leq L_{\mathrm{c}} \approx 1.5 L_{0}$, which is of the order of the minimum unstable wavelength of a flat sand bed [1]. The latter therefore also defines a minimum size for (steady-state) isolated heaps migrating over plane bedrock. Figure $2 \mathrm{~b}$ ) demonstrates that both $L_{0}$ and $L_{\mathrm{c}}$ are linearly related to $\ell_{\mathrm{s}}^{0}$.

The dependence of the critical height $H_{\mathrm{c}}$ and critical aspect ratio $H_{\mathrm{c}} / L_{\mathrm{c}}$ on the wind strength can be estimated analytically by observing that the flux over a longitudinal slice has to vanish upon creation of a slipface. This requires that the minimum shear stress at the feet of the heap, which deviates from $\tau^{0}$ by a factor proportional to $H / L$, equals $\tau_{\mathrm{t}}$ if $H=H_{\mathrm{c}}, L=L_{\mathrm{c}}$. Or, $H_{\mathrm{c}} / L_{\mathrm{c}} \propto 1-\tau_{\mathrm{t}} / \tau^{0}$, which is in good agreement with our numerical data (not shown) and mimicked by the aspect ratios of slices of fixed mass (figure 3a). By a similar argument [15] one can explain the affine dependence of the aspect ratio of a slice of given sand mass under a given wind speed on the influx (figure $3 \mathrm{~b}$ ). Note that contrary to the profiles in figures $2 \mathrm{a}$, $3 \mathrm{a}$, the profiles in figure $3 \mathrm{~b}$ are not in the steady state, but are slowly growing in time.

\section{Transverse profiles}

So far, we have discussed the profiles of longitudinal cuts through the dune, exclusively. To understand the transverse dune profile, the weak lateral coupling of adjacent longitudinal slices obviously has to be taken into account. In the spirit of a minimal description $\ddagger$, we regard as the conceptionally most important contributions lateral grain scattering in the hopping process and irregular lateral perturbations due to turbulence. Both effects already exist on a flat bed and give rise to lateral diffusion [17]. Though the neglected systematic slope effectsł cannot a priori be regarded as

$\ddagger$ In the minimal model [15], sand transport is modelled as on a flat sand bed, the topography enters only via its effect on the wind speed. Direct slope effects onto the flux (difference between uphill or downhill hopping, lateral deflection) and onto the local wind direction (wind deflection) are neglected. Due to the relatively mild slopes on the windward side, this is a good first approximation. 
small relative to this lateral diffusion, we prefer to neglect them here to keep the model 'minimal' and analytically tractable. Some investigations of how they affect the numerical solutions of the minimal model can be found in the literature $[9,21,23]$.

Within the mentioned restrictions, we make the phenomenologically plausible assumption that the lateral scattering is roughly proportional to the longitudinal flux $q_{\|}$itself, so that a net lateral flux will only occcur in response to lateral gradients in $q_{\|}$, i.e. $q_{\perp} \approx-\ell_{\perp} \partial q_{\|} / \partial y[17] \S$. The new phenomenological length scale $\ell_{\perp}$ can be expected to be a complicated function of the splash process, but will be proportional to (though supposedly numerically substantially smaller than) the average saltation length, i.e. the effective characteristic hopping length of the grains, which is predicted to be relatively weakly sensitive to changes in wind speed $[15,22]$ for the same reason as the grain speed. Therefore, $\ell_{\perp}$ will be treated as a constant phenomenological coefficient for the following considerations. We identify the spatial changes

$$
E_{y} \equiv \partial q_{\perp} / \partial y \approx-\ell_{\perp} \partial^{2} q_{\|} / \partial y^{2}
$$

in the lateral flux as a lateral erosion. From this we can deduce the transverse profile if we can relate the longitudinal flux to the height profile. Bagnold noticed [2] that for a steady-state dune with migration speed $v$, the ratio $q_{\|}^{\mathrm{b}}(y) / h^{\mathrm{b}}(y)$ of the sand flux over the brink to the height of the brink must be the same for any longitudinal slice $h_{y}(x)$ with slipface, or rather, $q_{\|}^{\mathrm{b}}(y)=\rho_{\mathrm{s}} v h^{\mathrm{b}}(y)$, where $\rho_{\mathrm{s}}$ is the density of the sand bed. Given that practically no sand is lost over the slipface, this immediately follows from mass conservation. Extending Bagnold's relation to regions somewhat upwind of the brink, we make the assumption that on vertical transverse slices $h_{x}(y)(x$ fixed) the longitudinal flux can still approximately be parametrized by

$$
q_{\|}(y) \approx \rho_{\mathrm{s}} v h_{x}(y) .
$$

Our conclusions would not change, though, if we allowed for additional terms $a_{x} y^{2}$, $b_{x} y^{4}$ with some $x$-dependent coefficients $a_{x}, b_{x}$ on the right hand side of this relation and/or terms $\tilde{a}_{x}, \tilde{b}_{x} y^{2}$ on the right hand side of equation (3).

We can now derive the profile of transverse slices $h_{x}(y)$ close to the crest of a large steady-state barchan dune from the requirement that the total lateral erosion and deposition (hence the square of $E_{y}$ ) be minimized for a given area of that slice,

$$
\delta \int \mathrm{d} y\left[\left(\partial^{2} q_{\|} / \partial y^{2}\right)^{2}+\mu h_{x}(y)\right]=0, \quad h_{x}( \pm Y)=0 .
$$

Here $Y(x)$ denotes the windward base-profile of the dune. Using equation (4), the solution for the transverse height profile is found to be a quartic polynomial that degenerates to a parabola

$$
h_{x}(y) \propto 1-(y / Y)^{2},
$$

in agreement with observations, if we require the slopes $\partial h_{x}(y) /\left.\partial y\right|_{Y}$ at the edges to be finite $[11,20] . \|$ This boundary condition guarantees that the otherwise eroded

$\S$ Because of the weak dependence of the average grain speed on the wind speed as a consequence of the non-trivial feedback of the mobilized grains onto the wind [15,22], this ansatz is essentially equivalent to a similar approach in reference [23] involving gradients in the density of saltating grains. Compared to the latter it represents a considerable simplification, because it does not oblige us to introduce separate dynamic equations for the density and velocity of the mobilized grains, respectively. || The remark after equation (4) gives some hint as to why some of the more complicated relations between the transverse and longitudinal flux proposed in the literature $[9,21,23]$ do not give rise to any significant numerical deviations of the transverse profile from the present result. Relaxing the steady-state assumption should also be uncritical. 
heap-like horns of the dune can be constantly rebuilt by the lateral flux. Since the total flux leaving the horns of width $w$ is of the order of $w q_{\mathrm{s}}^{0}$, conservation of horn mass requires $w\left(q_{\mathrm{s}}^{0} / 2-q_{\mathrm{i}}\right)=\int \mathrm{d} x q_{\perp \mathrm{h}}$, where $q_{\perp \mathrm{h}}$ is the $x$-dependent lateral flux onto a horn, i.e., $q_{\perp}$ taken at $y= \pm(W-w)$. For large dunes, $w \ll W$ and the integral is estimated as $2 \rho_{\mathrm{s}} v L \ell_{\perp} H / W$. Using $v \propto L^{-1}[14,15]$, we conclude that the transverse aspect ratio $H / W$ should, for given wind and influx conditions, not depend on dune size if the horn width $w$ does not, in accord with field observations [11,20].

\section{Conclusions}

We have presented evidence derived from the minimal model of aeolian sand dunes for similarity rules relating the profiles of dunes of different mass and under different environmental conditions. Many of the predictions are strongly supported by field measurements and by recent laboratory experiments. Some caution is needed, however, when applying the model to extreme conditions, e.g. to dunes on Mars. The general structure of the (hydrodynamic-type) model equations is expected to pertain to situations where particular predictions for their kinetic coefficients cease to apply. Our analysis suggests that both longitudinal and transverse vertical cuts through a barchan dune have universal profiles independent of dune mass, wind speed, and sand supply, because the scale invariance imposed by the turbulent wind is only weakly broken by the saturation transients in the sand flux. In general, the aspect ratios of the profiles change with these parameters, however. For the longitudinal cuts, the dependencies could be rationalized very well on the basis of the original dynamic equations of the minimal model, while a minimal, analytically tractable extension of the model was proposed for the transverse direction.

\section{References}

[1] B. Andreotti, P. Claudin, and S. Douady. Selection of dune shapes and velocities part 1: Dynamics of sand, wind and barchans. Eur. Phys. J. B, (28):321-339, 2002.

[2] R. A. Bagnold. The physics of blown sand and desert dunes. Methuen, London, 1941.

[3] A. Betat, V. Frette, and I. Rehberg. Sand ripples induced by water shear flow in an annular channel. Phys. Rev. Lett., 83:88-91, 1999.

[4] A. Betat, C.A. Kruelle, V. Frette, and I. Rehberg. Long-time behavior of sand ripples induced by water shear flow. Eur. Phys. J. E, 8:465-476, 2002.

[5] C. S. Breed. Terrestrial analogs of the Hellespontus dunes, Mars. Icarus, 30:326-340, 1977.

[6] K. S. Edgett and P. R. Christensen. The particle size of martian aeolian dunes. J. Geophys. Res., 96:22,765-22,776, 1991.

[7] N. Endo, K. Taniguchi, and A. Katsuki. Observation of the whole process of interaction between barchans by flume experiments. Geophys. Res. Lett., 31, 2004.

[8] Noritaka Endo, Hidehito Kubo, and Tsuguo Sunamura. Barchan-shaped ripple marks in a wave flume. Earth. Surf. Process. Landforms, 29:31-42, 2004.

[9] Pascal Hersen. On the crescentic shape of barchan dunes. Eur. Phys. J. B, 37:507-514, 2004.

[10] Pascal Hersen, Stéphane Douady, and Bruno Andreotti. Relevant length scale of barchan dunes. Phys. Rev. Lett., 89:264301, 2002.

[11] P. A. Hesp and K. Hastings. Width, height and slope relationships and aerodynamic maintenance of barchans. Geomorphology, 22:193-204, 1998.

[12] J. C. R. Hunt, S. Leibovich, and K. J. Richards. Turbulent wind flow over smooth hills. Q. J. R. Meteorol. Soc., 114:1435-1470, 1988.

[13] J. D. Iversen and B. R. White. Saltation threshold on Earth Mars and Venus. Sedimentology, 29:111-119, 1982.

[14] Klaus Kroy and Xiang Guo. Comment on "Relevant Length Scale of Barchan Dunes". Phys. Rev. Lett., 93:039401, 2004. 
[15] Klaus Kroy, Gerd Sauermann, and Hans J. Herrmann. Minimal model for aeolian sand dunes. Phys. Rev. E, 66:031302, 2002.

[16] Klaus Kroy, Gerd Sauermann, and Hans J. Herrmann. Minimal model for sand dunes. Phys. Rev. Lett., 88:054301, 2002.

[17] A.R. Lima, G. Sauermann, H.J. Herrmann, and K. Kroy. Modelling a dune field. Physica A, 310:487-500, 2002.

[18] J. R. Marshall, J. Borucki, and C. Bratton. Aeolian sand transport in the planetary context: respective roles of aerodynamic and bed-dilatancy thresholds. LPSC, XXIX:1131, 1998.

[19] P. R. Owen. Saltation of uniformed sand grains in air. J. Fluid. Mech., 20:225-242, 1964.

$[20]$ G. Sauermann, P. Rognon, A. Poliakov, and H. J. Herrmann. The shape of the barchan dunes of southern Morocco. Geomorphology, 36:47-62, 2000.

[21] Gerd Sauermann. Modeling of Wind Blown Sand and Desert Dunes. PhD thesis, Stuttgart Univ., 2001.

[22] Gerd Sauermann, Klaus Kroy, and Hans J. Herrmann. Continuum saltation model for sand dunes. Phys. Rev. E, 64:031305, 2001.

[23] V. Schwämmle and H. Herrmann. A model of barchan dunes including lateral shear stress. cond-mat/0304695, may 2003.

[24] A. Shields. Anwendung der Ähnlichkeitsmechanik und der Turbulenzforschung auf die Geschiebebewegung. Mitteilungen der Preuss. Versuchsanst. f. Wasserbau u. Schiffbau, Berlin, 26, 1936.

[25] P. C. Thomas, M. C. Malin, M. H. Carr, G. E. Danielson, M. E. Davies, W. K. Hartmann, A. P. Ingersoll, P. B. James, A. S. McEwen, L. A. Soderblom, and J. Veverka. Bright dunes on Mars. Nature, 397:592-594, 1999.

[26] W. S. Weng, J. C. R. Hunt, D. J. Carruthers, Warren A., G. F. S. Wiggs, I. Livingstone, and I. Castro. Air flow and sand transport over sand-dunes. Acta Mech. Suppl., 2:1-22, 1991. 